\title{
Fragility fractures of the pelvis
}

\author{
Gillian L. S. Soles • Tania A. Ferguson
}

Published online: 16 May 2012

(C) Springer Science+Business Media, LLC 2012

\begin{abstract}
Fragility fractures of the pelvis are common and the incidence is increasing with the aging population. The primary risk factor is osteoporosis. Diagnosis is challenging and advanced imaging with computed tomography (CT), bone scintigraphy, and magnetic resonance imaging (MRI) is helpful. These injuries result in significant morbidity, including prolonged hospitalization, immobility, and loss of autonomy in previously active patients. The mortality rate is high, similar to hip fracture patients. This problem is underappreciated and deserves attention. An opportunity exists to improve outcomes with medical and surgical management.
\end{abstract}

Keywords Fragility fractures · Sacral insufficiency fractures $\cdot$ Osteoporotic fractures $\cdot$ Elderly pelvic ring fractures $\cdot$ Trauma

\section{Introduction}

"Fragility fractures" of the hip, vertebrae, and wrist are widely recognized. These fractures typically result from a low energy trauma such as a fall. Severely osteoporotic bone can fail simply due to lack of osseous architecture and these "insufficiency" fractures may occur in the absence of trauma. Fractures afflicting the osteoporotic pelvis have recently gained interest due to their increasing incidence, which

\section{G. L. S. Soles $\cdot$ T. A. Ferguson $(\bowtie)$}

Department of Orthopaedic Surgery, UC Davis Medical Center, 4860 Y Street Suite 3800,

Sacramento, CA 95817, USA

e-mail: tania.ferguson@ucdmc.ucdavis.edu

G. L. S. Soles

e-mail: gillian.soles@ucdmc.ucdavis.edu parallels the age of the general population. Many of these patients suffer fragility fractures as a result of a fall from standing, while others present with assumed insufficiency fractures of the sacrum and report no traumatic event.

These fractures deserve attention as they lead to a decrease in mobility with an increase in dependency, and are associated with a high rate of mortality $[1 \bullet, 2,3,4 \bullet]$. We aim to deepen the understanding of the epidemiology, risk factors, mechanism of injury, treatment, and outcomes of osteoporotic pelvic fractures.

\section{Epidemiology}

Fragility fractures of the pelvis (fractures occurring in patients $>65$ years of age after low energy falls) are remarkably common. Pelvic fractures represent $7 \%$ of all osteoporosis related fractures in people $\geq 50$ years of age in the United States, and account for $5 \%$ of the total cost burden [5]. Although there is more published data on high-energy unstable pelvic ring injuries in young patients, Kannus et al point out that the majority of pelvic fractures today have an osteoporotic origin. They note that $64 \%$ of all pelvic fractures are osteoporotic fractures, and in patients age $>60$ years, the percentage increases to $94 \%\left[6^{\bullet}\right]$.

The incidence of pelvic insufficiency fractures is increasing and is age-related. Melton et al from the Mayo Clinic reported on the incidence of pelvic fractures over a ten-year period (1968 to 1977). The incidence of pelvic fractures sustained via low energy trauma, such as a simple fall from standing, rose exponentially in both men and women as they aged. For men aged 55-74, the incidence of low energy pelvic fractures was $7 / 100,000$ person-years. This rate increased to $63.9 / 100,000$ person-years in men 75 years and older and to $220.3 / 100,000$ person-years in those older than 
85. For women, the rate increased from $56.9 / 100,000$ person-years for the age group $55-74$, to $249.5 / 100,000$ person-years in those 75 and older, and to $446.3 / 100,000$ person-years for women over the age of 85 [7]. Kannus et al reported a similar increase experienced in Finland. These authors report that the incidence of osteoporotic pelvic fractures has increased an average of $23 \%$ per year over the last three decades for both men and women $>60$ years of age and $54 \%$ per year in women $>80$ years of age. Based on data from the last 30 years, they predict that the current number of osteoporotic pelvic fractures will triple by the year 2030 $[6 \bullet]$. A combination of a true increase in incidence as well as an increase in awareness with improvements in diagnosis may be contributing to these reports; however, the alarming numbers point to an important public health care problem.

\section{Risk factors}

Patients with pelvic insufficiency fractures have an underlying diagnosis of osteoporosis [8]. In general, the difference between patients with sacral insufficiency fractures and hip fractures is the severity of osteoporosis. Of 115 patients with pelvic fractures evaluated by Morris et al, $93 \%$ had a Singh index of four or less, consistent with osteoporosis [4•]. This is compared to $67.9 \%$ of patients with femoral neck fractures and $8.9 \%$ of controls in a study by Pogrund et al [9].

Prior internal fixation of the proximal femur and hip arthroplasty are risk factors for pelvic insufficiency fractures. These factors often indicate osteoporosis and prior hip fracture, both of which are independent risk factors for future insufficiency fracture. Also, a fall onto a previously stabilized or replaced hip results in injury force transmission to the pelvis and sacrum, resulting in a "lateral compressive" injury (see below for fracture patterns). Both lower lumbar arthritis and scoliosis have been associated with sacral insufficiency fractures and sacral nonunions. Gotis - Graham reported on 20 patients with sacral insufficiency fractures. Seven of them had lumbar scoliosis, with the sacral fracture opposite the convexity of the curve in five of these patients [10]. We postulate that scoliosis creates an iliac prominence such that the fall from standing results in impact to the ilium (rather than the greater trochanter), and a lateral compressive type pelvic injury (rather than hip fracture). Lumbar spine stiffness secondary to arthritis results in force transmission to the upper sacral segments, increasing the motion experienced in this region in a way that may be detrimental to fracture healing.

\section{Fracture classification}

Pelvic insufficiency fractures are the result of normal physiologic stress on abnormally weak bone and do not always fit into traditional classification schema. They are almost exclusively sacral fractures and can be further divided by Denis [11]. Furthermore, there are a disproportionately high number of cases that cross the midline and fall into the " $\mathrm{H}$ " or "U" shaped categories.

Using the classification of Young and Burgess [12], many of these injuries appear as lateral compression (LC) injuries. In fact, LC injuries are five times more common than anterior - posterior compression (APC) injuries in the elderly [13]. Typically the patient falls from standing onto their side, resulting in a laterally based force on the pelvis. A compression fracture to the ipsilateral sacrum occurs, the hemipelvis internally rotates, and the pubic and ischial rami fracture in a "horizontal" nature. The internal rotational displacement/deformity is rarely severe in the elderly patients, and the diagnosis is made based on the radiographic appearance of the rami fractures, as the sacral fracture is very difficult to appreciate radiographically.

A vertical shear injury pattern is often recognized as well. An axial stress, if the patient falls directly on their coccyx/ sacrum, or simply "sits too hard," results in unilateral or commonly bilateral sacral fractures that run parallel to the SI joints in a "vertical" pattern. When bilateral, these fractures commonly "connect" with a horizontal injury resulting in an " $H$ " or " $U$ " shaped appearance $[14,15]$. In a large anatomical and biomechanical study of sacral insufficiency fractures, the most common pattern identified was the " $\mathrm{H}$ " shaped pattern with bilateral vertical Denis Zone I sacral fractures along with a transverse component, crossing through the lower portion of S1 or upper portion of S2 [16]. Multiple authors have recognized this pattern, citing it is diagnostic of sacral insufficiency fractures [17-20]. These fractures may occur with any combination of ramus fractures (unilateral, bilateral) and may present without an anterior ring injury. Although these fractures can present without notable deformity, one must always check for sagittal plane displacement in the presence of bilateral sacral injuries. It is not uncommon for these injuries to be missed on the AP pelvis with a typical kyphotic or lordotic displacement through the transverse fracture (seen only on the sagittal CT reconstructions), which can result in cauda equina syndrome.

\section{Mechanism of injury}

Low energy falls are responsible for the majority of pelvic insufficiency fractures; however, up to two-thirds have been noted to occur in the absence of trauma [8]. In a large series of consecutive elderly patients with osteoporotic ramus fractures, Hill et al reported the mechanism of injury as simple falls in $87.4 \%$ of the cases, with over half of these falls occurring at home [1•]. Similarly, Morris et al noted that $83 \%$ of the closed pelvic fractures in their series were 
the result of falls from standing, walking, or transferring [4•]. Simple falls also accounted for $86.6 \%$ of the pelvic insufficiency fractures studied by Taillandier [2] and $89 \%$ of the pelvic fractures studied by Breuil [3]. Many elderly patients sustain femoral neck or intertrochanteric hip fractures as a result of falls, but many patients who sustain fragility fractures have previously fractured their hips and have implants protecting the proximal femur.

\section{Diagnostic imaging}

All patients presenting with pelvic or sacral pain should undergo thorough radiographic evaluation. AP, inlet, outlet, and Judet radiographs are appropriate for initial evaluation of the pelvis and acetabulum. The pelvic films can be especially difficult to interpret. As is the case for young patients, overlying bowel gas and bladder contrast commonly obscure visibility of the sacrum and posterior pelvis. The diagnosis of the insufficiency fracture is especially challenging with plain films, due to the osseous demineralization associated with the severe osteoporosis, as well as the general lack of displacement associated with these injuries (Fig. 1a). For these reasons, only $20-38 \%$ of sacral insufficiency fractures are identified on plain radiographs, and many of those are missed on initial evaluation [21]. Frequently, the pubic rami fractures are the only observed disruption. However, studies have shown these fractures do not occur in isolation [22], and when noted in the elderly patient, further imaging should be performed to look for the sacral injury. We recommend advanced imaging of any patient presenting with severe osteoporosis, pelvic pain (with or without trauma), and inconclusive or negative pelvic radiographs.

Bone scintigraphy with technetium-99m medronate methylene diphosphonate (Tc-99m-labeled MDP) has a reported sensitivity of $96 \%$ and positive predictive value of $92 \%$ [21]. The classic " $H$ " shaped pattern may be visualized with this imaging modality. As noted in a metaanalysis by Finiels et al, this classic " $\mathrm{H}$ " shaped pattern is evident in about $42.7 \%$ of cases [23]. Multiple variations have been reported including bilateral alar uptake with a partial horizontal bar, bilateral alar uptake, unilateral alar uptake with a horizontal bar, unilateral alar uptake, and a horizontal linear dot pattern in the inferior sacral body [19]. In a case review by Gotis-Graham et al, bone scans were positive in the entire cohort of 20 patients, while CT demonstrated a fracture or evidence of sclerosis in seven of 12 patients, and plain radiographs demonstrated sclerosis in only four of 20 patients [10]. Peh et al reported positive bone scans in 21 of 22 patients with documented sacral insufficiency fractures, while CT identified fractures in only seven of 22 patients [24].
The diagnostic capacity of CT has improved markedly over the last two decades and, in our practice, is used to further evaluate any patient suspected of having a sacral insufficiency fracture. Fine-cut axial CT imaging with coronal and sagittal reconstructions aids in visualizing the full extent of fracture lines, the osseous architecture, and the displacement (Fig. 1b, c). Subtle injuries to the posterior pelvic ring missed on plain radiographs can be more clearly identified. Vertical fracture lines and bony sclerosis may be seen parallel to the sacroiliac (SI) joints. The horizontal component to the fracture, often seen along with the vertical fracture lines that create the classic " $H$ " shaped pattern, is best seen on the coronal reconstruction. The sagittally reconstructed images ("lateral") are critical in evaluating these injuries to identify the typical dorsal or ventral displacement that can occur and cause cauda-equina syndrome. In addition, CT scans help to differentiate insufficiency fractures from metastatic disease, primary bone or soft tissue tumors, or osteomyelitis, all of which give the same appearance with scintigraphy.

MRI has a similar sensitivity to bone scans and can detect sacral insufficiency fractures soon after symptoms develop. On standard MRI evaluation of sacral insufficiency fractures, $\mathrm{T} 1$ images demonstrate low signal intensity while T2 images demonstrate high signal intensity, consistent with marrow edema. The imaging sequences most sensitive for the detection of marrow edema associated with sacral insufficiency fractures are T2 short tau inversion recovery (STIR) sequences and T2 images with fat suppression [20]. Marrow edema caused by sacral insufficiency fractures can usually be differentiated from that caused by metastatic disease. Cabarrus et al evaluated the ability of MRI and CT to detect insufficiency fractures of the pelvis and proximal femur. Known sacral insufficiency fractures were identified in only 50 of 67 patients $(74.6 \%)$ using CT scans, while MRI detected $100 \%$. Similarly, $65.5 \%$ of pubic fractures were found using CT scans, while MRI again detected $100 \%$ [25]. In a series of 50 consecutive elderly patients with pubic rami fractures, Cosker et al evaluated the pelvic ring with MRI and 45 of the 50 patients had associated vertically-oriented compression fractures of the sacrum [26]. In the majority of cases, the pubic rami fracture and sacral injury were ipsilateral. This is consistent with common patterns of injury. With a sensitivity approaching that of bone scans, MRI is helpful in the diagnosis of sacral insufficiency fractures.

In our practice, we find that plain films are important in the initial work-up of patients with osteoporosis and pelvic pain; however, plain radiography is never sufficient to fully understand the injury, and many injuries are missed without advanced studies. CT scanning is part of our routine radiographic protocol in the diagnosis of pelvic insufficiency fractures and includes $1.3 \mathrm{~mm}$ axial cut acquisition, and 
Fig. 1 Case example. An 87 year-old female, with history of bilateral hip fractures treated with sliding hip screws, and vertebral compression fractures treated with kyphoplasty, who sustained a simple fall and presented with low back and pelvic pain. Panel a: AP pelvis on presentation. Note the sacral fracture is extremely difficult to appreciate on this radiograph. Panel b: Axial CT. Note severe osteoporosis throughout the sacrum. Panel c: Sagittal CT. Note kyphosis at the level of S1-S2 indicating the horizontal component of this Ushaped fracture. Panel d: Postoperative AP pelvis. Transsacral screws were placed at S1 and S2 and augmented with calcium phosphate cement at the sacral fracture sites bilaterally
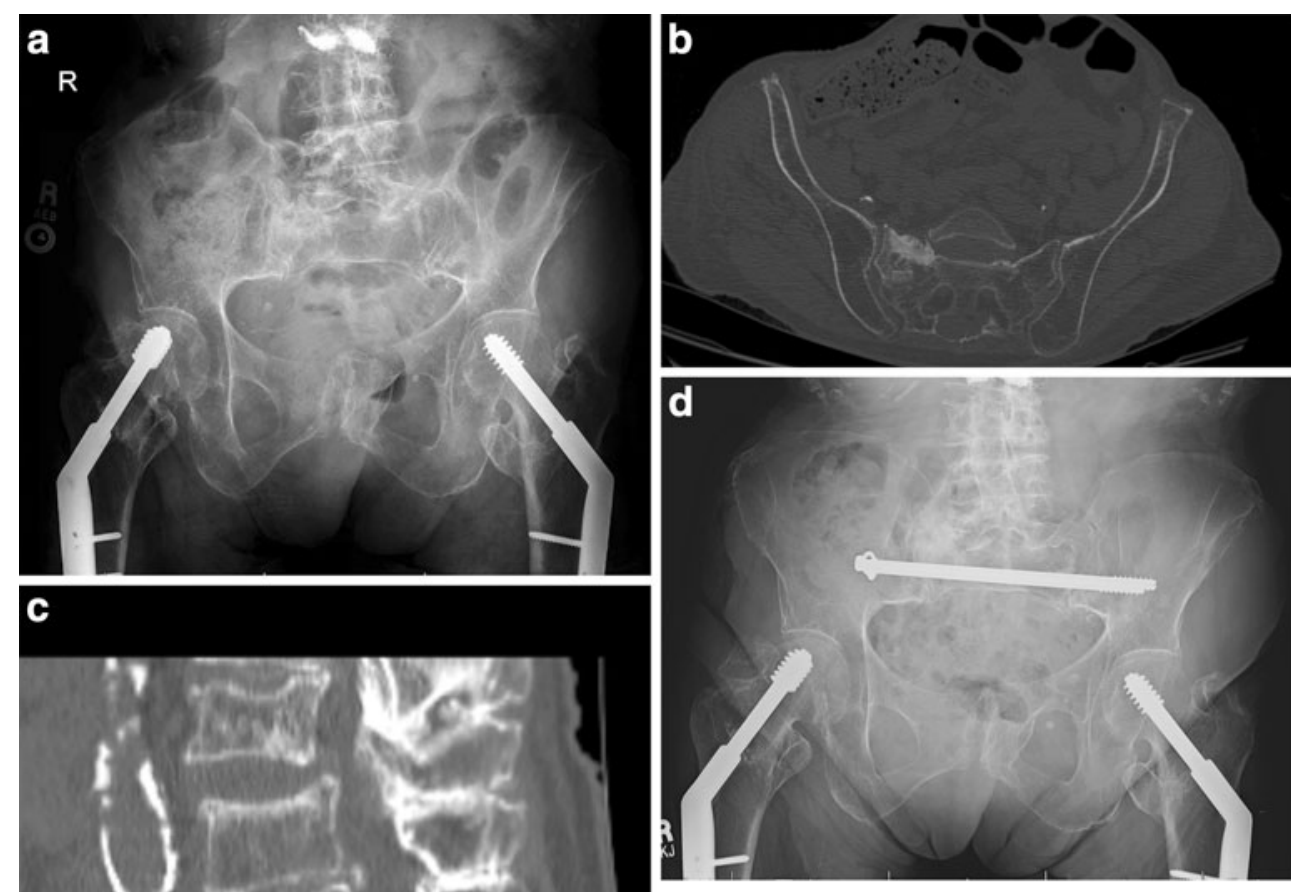

coronal and sagittal plane reconstructed images. If the initial protocol does not identify an injury, we proceed to MRI scanning, which is highly sensitive and specific. We have replaced bone scans with CT and MRI, which together are highly diagnostic, give information regarding the osseous anatomy, and allow for surgical planning when considered.

\section{Nonoperative treatment and outcomes}

Currently, nonoperative management consists of rest, analgesia, and physical rehabilitation and this form of treatment, or lack thereof, is reflected in the outcomes reported.

Immobility has been associated with serious complications; notably, decreased muscle strength, deep venous thrombosis (DVT) and pulmonary embolism (PE), postural hypotension, decreased cardiac function, urinary retention and calculus formation, constipation, pressure ulcers, impaired pulmonary function resulting in pneumonia, and bone resorption with worsening osteoporosis. In addition to this physiologic dysfunction, immobility precipitates psychologic dysfunction including anxiety and depression[27].
The average number of days in the hospital following sacral insufficiency fracture is high. Across several series, the mean acute inpatient stay ranged from 9.3 to 45 days, with a minimum of one day and a maximum of 130 days $[1 \bullet$, $2,3,4 \bullet]$. The length of stay was longer for patients who were not self-sufficient prior to fracture [2].

Patient autonomy is significantly impacted by sacral insufficiency fractures. There are several reports suggesting that patients experience a minimal change in independence and have limited pain following sacral insufficiency fractures $[10,28]$. However, most published series indicate a significant change in post-injury ambulatory status as well as chronic pain after conservative management of these injuries. Hill et al reported on the five-year survival in a series of 286 consecutive patients with pubic rami fractures. At final follow up, 139 patients were still living and only $51.1 \%$ were independently mobile, while $38.8 \%$ were using walking aids and $10.1 \%$ were wheelchair bound or immobile [1•]. This decline in mobility had a commensurate increase in social dependency. Morris et al also found a significant impact on the mobility status and dependency following pelvic fracture in a series of 148 patients over age 
65. At the time of hospital discharge, 137 patients were still living; none were able to walk independently;, $48.9 \%$ required the use of an aid, and $51.1 \%$ required physical assistance. The $70.9 \%$ of patients able to return to their previous living situation required the support of social services in $84.3 \%$ of cases. The $29.1 \%$ unable to return to their previous living situation became institutionalized[4•]. Of 60 patients over age 65 reviewed by Tallandier et al, 56 had outcome data available at one year. Only 22 patients returned to their baseline functional status, while ten experienced a decrease in function and seven patients died [2]. In another series of 60 patients over age 65 evaluated by Breuil et al, only $31 \%$ of patients returned to their own home at the time of discharge, while $65.6 \%$ required ongoing inpatient care, and $3.4 \%$ were permanently institutionalized. Data was available for 51 of these patients at a mean follow up of 29 months (range 2-58 months) and a loss of autonomy was found in nearly $50 \%$ of the patients [3]. Previously active, independent patients suffer major functional impairment as a result of sacral insufficiency fractures.

The mortality rate of patients with sacral insufficiency fractures is high, similar to hip fractures. In the series by Hill et al, the inpatient mortality rate was $7.0 \%$. The overall mortality rate at one year was $13.3 \%$ and climbed to $54.4 \%$ at five years. At the five year follow-up, there was no difference in mortality between patients with pelvic fractures and hip fractures [1•]. Morris et al reported a similar inpatient mortality rate of $7.6 \%$. More striking, however, was the one year mortality rate of $27 \%$ with an annual increase of $10 \%$, resulting in a three year mortality rate of $50 \%$ [4 ]. Breuil et. reported mortality in $37.5 \%$ of men and $18.6 \%$ of women at an average of 29 months following fracture [3].

Contemporary literature demonstrates that sacral insufficiency fractures result in decreased mobility, increased physical and social dependency, and a high rate of morbidity and mortality. We feel strongly that a more aggressive medical and surgical intervention program can improve these dismal outcomes.

\section{Medical management}

Osteoporosis is the primary risk factor for sacral insufficiency fractures and medical management consists of supplementation with calcium and vitamin $\mathrm{D}$, along with antiresorptive or anabolic agents to prevent additional fractures.

Bisphosphonates are antiresorptive agents that inactivate osteoclastic bone resorption, decreasing bone turnover and bone loss [29]. Studies have shown bisphosphonate use results in increased bone mineral density in the spine and hip and decreased incidence of new fractures in patients with osteoporosis [30, 31]. However, these drugs are not without complications, including gastrointestinal discomfort, acute influenza-like illness, renal insufficiency, osteonecrosis of the jaw, and atypical stress fractures.

Recently, attention has been turned to the use of anabolic agents, such as recombinant human parathyroid hormone (PTH), to increase bone mineral density, reduce fracture rate, and improve fracture healing. In a randomized controlled trial, Peichl et al compared PTH 1-84 with placebo in elderly patients with osteoporotic pelvic fractures with a primary end point of fracture healing. PTH 1-84 represents the full-length peptide, as compared to teriparatide or forteo, which is PTH 1-34. Patients with a unilateral pelvic fracture, who were greater than 70 years of age, and who met the WHO definition for osteoporosis with a T-score $<-2.5$ at the lumbar spine or proximal femur, were included. All patients received supplementation with $1,000 \mathrm{mg}$ of calcium and 800 IU of vitamin D3 daily. In the PTH 1-84 group, fracture healing was noted at 7.8 weeks as compared to 12.6 weeks in the control group ( $p<0.001$ ). Healing was based on serial CT scans obtained at $0,4,8,12$ weeks and at regular intervals until evidence of cortical bridging was noted. The authors conclude that PTH 1-84 can be used to accelerate fracture healing, which may aid in pain relief and patient mobilization, thus decreasing complications related to immobility [32•].

Medical management is an important component in the treatment of osteoporotic fractures. For the at-risk elderly population, patients with a diagnosis of osteoporosis, and patients with known sacral insufficiency fractures, supplementation with calcium and vitamin $\mathrm{D}$ is recommended. The emerging evidence evaluating the role of recombinant PTH in the setting of insufficiency fractures is encouraging. Based on the reported success of recombinant PTH, further research on its clinical applications, effect on bone mineral density, fracture risk, and fracture healing is warranted.

\section{Surgical treatment}

Sacroplasty, injection of polymethylmethacrylate (PMMA) into the sacral fracture, has been applied to pelvic insufficiency fractures. This technique has been used in osteoporotic vertebral compression fractures, and is now being applied to the sacrum and, to a lesser extent, the ilium and ramus. The thought is that pain and dysfunction are related to micro motions at the fracture site, and augmentation with PMMA may provide mechanical stabilization and therefore pain relief.

There are several small, retrospective case series reporting clinical success with sacroplasty for fractures. Pommersheim et al [33] reported on three patients and Butler et al [34] reported on four patients. Both lack meaningful follow- 
up, but reported improvement in patient symptoms. Frey et al reported on a cohort of 37 patients followed for one year and noted improvement in the immediate visual analog scale (VAS) for pain and decreased opioid use with percutaneous sacroplasty [35]. This study is the only published report available with a meaningfully sized cohort, but lacks detail about the cohort's injuries and data collection methodology. Furthermore, given the lack of a control group, these results should be interpreted with caution.

Complications associated with this technique relate to extravasation of the PMMA into the pre-sacral space, spinal canal, sacral foramen, or SI joints, and may result in sacral nerve root or sacral spinal canal compromise, or SI joint dysfunction. Furthermore, a biomechanical analysis by Richards et al demonstrated that sacroplasty does not restore the strength or stiffness of the sacrum, regardless of the volume or location of the cement [36].

Pelvic surgeons have an opportunity to improve the outcomes of these fractures over the current standard of management. Basic principles of fracture management, including mechanical stabilization, fracture compression, and bone defect management, should be employed.

Matta et al reported on transsacral screw fixation of the posterior pelvic ring, noting that the greater screw length provided more secure fixation, protection against vertical shear stress, and biomechanically sound fracture compression[37]. Gardner and Routt echo these thoughts in their case series, which included patients with osteoporosis and insufficiency fractures [38•]. Particularly in osteoporotic patients, when the sacral body and ala have minimal osseous composition for screw purchase, the screw threads of a long trans-sacral screw placed into the contra-lateral SI joint may allow improved fixation and decreased likelihood of pullout. This has become the mainstay of our surgical management of these patients (Fig. 1d).

Even with trans-sacral screws, fracture compression poses a significant challenge. The ability to generate compression is dependant on the contra-lateral SI joint and ilium, and in the most severe cases, is insufficiently strong to prevent the screw from stripping as one tightens the head. The transsacral screw technique was modified by Moed et al to include a locking mechanism on the far side of the screw. These authors conclude this is beneficial for bilateral posterior ring injuries as well as in severe pelvic osteopenia, as the locked end prevents screw pullout, regardless of purchase [39]. We create locking bolts with $1 \times 1 \mathrm{~cm}$ femoral struts that the $8.5 \mathrm{~mm}$ screw threads into to create the same effect. With improved thread fixation following one of these techniques, one must take caution not to overtighten the screw to avoid intrusion of the washer into the ipsilateral ilium.

Our current practice is to offer surgery to patients with either severe pain from acute fractures or non-union/delayed union appreciated in the outpatient setting. Our approach includes an emphasis on fracture reduction in the setting of displacement, mechanical stabilization with trans-sacral lag screw fixation (locked with femoral struts as needed,) and augmentation with either allograft, autograft, or calciumbased bone cements in the setting of severe sacral osseous insufficiency (Fig. 1d).

\section{Conclusions}

The elderly population is rapidly growing and the burden of osteoporosis and fragility fractures is a problem faced by physicians and surgeons across disciplines. The primary risk factor for fragility fractures of the pelvis is osteoporosis. We should maintain a high index of suspicion for sacral insufficiency fractures in elderly patients presenting with low back or pelvic pain following simple falls or little to no trauma, and pelvic X-rays should be accompanied with a CT scan. Advanced imaging with bone scan or MRI is reserved for patients with pain and negative CT scans. The " $\mathrm{H}$ " shaped pattern of injury is pathognomonic.

Outcomes of conservative management are poor, with decreased mobility, increased physical and social dependency, and high mortality. All patients should be treated for the severe osteoporosis associated with this injury, and the use of recombinant PTH should be further evaluated. The initial reports of this treatment are very encouraging. Surgical intervention should be further studied and has the potential to allow immediate weightbearing, thus limiting the adverse events associated with immobility and decreasing the acute and chronic pain associated with these fractures.

Disclosure No potential conflicts of interest relevant to this article were reported.

\section{References}

Papers of particular interest, published recently, have been highlighted as:

- Of importance

1. - Hill RM, Robinson CM, Keating JF. Fractures of the pubic rami. Epidemiology and five-year survival. J Bone Joint Surg Br. 2001;83(8):1141-4. This study points out the high rate of mortality associated with osteoporotic pelvic fractures. In addition, at five years there was no difference in mortality between patients with pelvic fractures and patients with hip fractures. We feel this finding is important and should be emphasized.

2. Taillandier J, Langue F, Alemanni M, et al. Mortality and functional outcomes of pelvic insufficiency fractures in older patients. Joint Bone Spine. 2003;70(4):287-9. 
3. Breuil V, Roux CH, Testa J, et al. Outcome of osteoporotic pelvic fractures: An underestimated severity. Survey of 60 cases. Joint Bone Spine. 2008;75(5):585-8.

4. - Morris RO, Sonibare A, Green DJ, et al. Closed pelvic fractures: characteristics and outcomes in older patients admitted to medical and geriatric wards. Postgrad Med J. 2000;76(900):646-50. This study is important to our understanding of the poor patient outcomes following conservative management of sacral insufficiency fractures. The authors demonstrate a significant impact on patient mobility and dependence post injury.

5. Burge R, Dawson-Hughes B, Solomon DH, et al. Incidence and Economic Burden of Osteoporosis-Related Fractures in the United States, 2005-2025. J Bone Miner Res. 2006;22(3):465-75.

6. - Kannus PP, Palvanen MM, Niemi SS, et al. Epidemiology of osteoporotic pelvic fractures in elderly people in Finland: sharp increase in 1970-1997 and alarming projections for the new millennium. Osteoporos Int. 2000;11(5):443-8. This epidemiologic study highlights the rapid increase in the incidence of osteoporotic pelvic fractures in the elderly patient population. The authors emphasize that this trend is expected to continue with the number of fractures tripling by 2030. This data points to an important public health problem which deserves our attention..

7. Melton LJ, Sampson JM, Morrey BF, et al. Epidemiologic features of pelvic fractures. Clin Orthop Relat Res. 1981;155:43-7.

8. Tsiridis E, Upadhyay N, Giannoudis PV. Sacral insufficiency fractures: current concepts of management. Osteoporos Int. 2006;17 (12):1716-25

9. Pogrund H, Rigal W, Makin M, et al. Determination of osteoporosis in patients with fractured femoral neck using the Singh index: a Jerusalem study. Clin Orthop Relat Res. 1981;156:189.

10. Gotis-Graham I, McGuigan L, Diamond T, et al. Sacral insufficiency fractures in the elderly. J Bone Joint Surg Br. 1994;76 (6):882.

11. Denis F, Davis S, Comfort T. Sacral fractures: an important problem. Retrospective analysis of 236 cases. Clin Orthop Relat Res. 1988;227:67-81.

12. Dalal S, Burgess A, Siegel J, et al. Pelvic fracture in multiple trauma: classification by mechanism is key to pattern of organ injury, resuscitative requirements, and outcome. J Trauma. 1989;29(7):981-1000. discussion 1000-2.

13. Krappinger D, Kammerlander C, Hak DJ, et al. Low-energy osteoporotic pelvic fractures. Arch Orthop Trauma Surg. 2010;130 (9):1167-75.

14. Roy-Camille R, Saillant G, Gagna G, et al. Transverse fracture of the upper sacrum. Suicidal jumper's fracture. Spine. 1985;10 (9):838-45.

15. Sabiston C, Wing P. Sacral fractures: classification and neurologic implications. J Trauma. 1986;26(12):1113-5.

16. Linstrom NJ, Heiserman JE, Kortman KE, et al. Anatomical and Biomechanical Analyses of the Unique and Consistent Locations of Sacral Insufficiency Fractures. Spine. 2009;34(4):309-15.

17. De Smet AA, Neff JR. Pubic and sacral insufficiency fractures: clinical course and radiologic findings. AJR Am J Roentgenol. 1985;145(3):601-6.

18. Grasland A, Pouchot J, Mathieu A, et al. Sacral insufficiency fractures: an easily overlooked cause of back pain in elderly women. Arch Intern Med. 1996;156(6):668-74.

19. Peh WC, Khong PL, Yin Y, et al. Imaging of pelvic insufficiency fractures. Radiographics. 1996;16(2):335-48.

20. Blake SP. Sacral insufficiency fracture. Br J Radiol. 2004;77 (922):891-6.

21. Lyders EM, Whitlow CT, Baker MD, et al. Imaging and Treatment of Sacral Insufficiency Fractures. Am J Neuroradiol. 2010;31 (2):201-10.

22. Gertzbein SD, Chenoweth DR. Occult injuries of the pelvic ring. Clin Orthop Relat Res. 1977;128:202-7.
23. Finiels H, Finiels P, Jacquot J, et al. Fractures of the sacrum caused by bone insufficiency. Meta-analysis of 508 cases. Presse Med. 1997;26(33):1568-73.

24. Peh W, Khong P, Ho W, et al. Sacral insufficiency fractures. Spectrum of radiological features. Clin Imaging. 1995;19(2):92101.

25. Cabarrus MC, Ambekar A, Lu Y, et al. MRI and CT of Insufficiency Fractures of the Pelvis and the Proximal Femur. Am J Roentgenol. 2008;191(4):995-1001.

26. Cosker TDA, Ghandour A, Gupta SK, et al. Pelvic ramus fractures in the elderly. Acta Orthop. 2005;76(4):513-6.

27. Babayev M, Lachmann E, Nagler W. The controversy surrounding sacral insufficiency fractures: to ambulate or not to ambulate? Am J Phys Med Rehabil. 2000;79(4):404-9.

28. Koval KJ, Aharonoff GB, Schwartz MC, et al. Pubic rami fracture: A benign pelvic injury? J Orthop Trauma. 1997;11(1):7.

29. Rodan GA, Fleisch HA. Bisphosphonates: mechanisms of action. J Clin Invest. 1996;97(12):2692.

30. Reginster J, Minne H, Sorensen O, et al. Randomized trial of the effects of risedronate on vertebral fractures in women with established postmenopausal osteoporosis. Vertebral Efficacy with Risedronate Therapy (VERT) Study Group. Osteoporos Int. 2000;11(1):83-91.

31. Liberman U, Weiss S, Broll J, et al. Effect of oral alendronate on bone mineral density and the incidence of fractures in postmenopausal osteoporosis. The Alendronate Phase III Osteoporosis Treatment Study Group. N Engl J Med. 1995;333(22):1437-43.

32. - Peichl P. Parathyroid Hormone 1-84 Accelerates FractureHealing in Pubic Bones of Elderly Osteoporotic Women. J Bone Joint Surg Am. 2011;93(17):1583. This randomized controlled trial evaluating the use of recombinant human PTH in insufficiency fractures has encouraging results. The data suggests that recombinant human PTH can increase bone mineral density, reduce fracture risk, and enhance fracture healing. Further research is needed to determine the precise role of recombinant human PTH in the medical management of insufficiency fractures. .

33. Pommersheim W, Huang-Hellinger F, Baker M, et al. Sacroplasty: a treatment for sacral insufficiency fractures. Am J Neuroradiol. 2003;24(5):1003-7.

34. Butler CL, Given CA, Michel SJ, et al. Percutaneous sacroplasty for the treatment of sacral insufficiency fractures. AJR Am J Roentgenol. 2005;184(6):1956-9.

35. Frey ME, DePalma MJ, Cifu DX, et al. Efficacy and safety of percutaneous sacroplasty for painful osteoporotic sacral insufficiency fractures: a prospective, multicenter trial. Spine. 2007;32 (15):1635-40.

36. Richards AM, Mears SC, Knight TA, et al. Biomechanical Analysis of Sacroplasty: Does Volume or Location of Cement Matter? Am J Neuroradiol. 2008;30(2):315-7.

37. Beaulé PE, Antoniades J, Matta JM. Trans-sacral fixation for failed posterior fixation of the pelvic ring. Arch Orthop Trauma Surg. 2005;126(1):49-52.

38. - Gardner MJ, Routt MLC. Transiliac-transsacral screws for posterior pelvic stabilization. J Orthop Trauma. 2011;25(6):378-84. In this case series including patients with osteoporosis and insufficiency fractures the surgical technique and clinical experience using transiliac-transsacral screws is reported. The important points to note are greater screw length assists in distributing the load and resisting vertical shear forces, the holding power is increased by the additional screw threads, and that anchoring the screw in iliac cortical bone aids in maintenance of reduction. This technique is the mainstay of our surgical management of these patients.

39. Moed BR, Whiting DR. Locked transsacral screw fixation of bilateral injuries of the posterior pelvic ring: initial clinical series. J Orthop Trauma. 2010;24(10):616. 